\title{
SDH Complex Mutation Analysis Was Not Performed
}

National Cancer Institute

\section{Source}

National Cancer Institute. SDH Complex Mutation Analysis Was Not Performed. NCI

Thesaurus. Code C160498.

An indication that SDH complex mutation analysis was not performed during the study. 